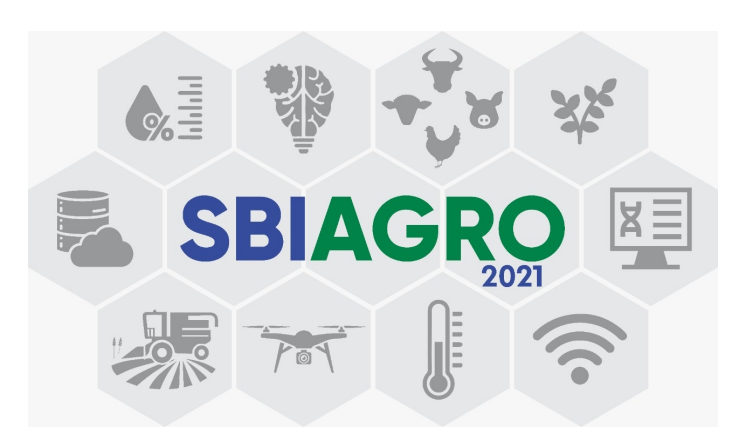

\title{
H-Pasture: uma aplicação móvel para estimativa da disponibilidade de forragem e ajuste de carga em pastagens sob lotação contínua ou rotativa
}

\author{
Wendell Gasparoni $^{1}$, Ana Paula Lüdtke Ferreira ${ }^{1}$, \\ Teresa Cristina Moraes Genro ${ }^{2}$, Márcia Cristina Teixeira Silveira ${ }^{2}$ \\ ${ }^{1}$ Universidade Federal do Pampa \\ Bagé - RS - Brasil \\ ${ }^{2}$ EMBRAPA Pecuária Sul \\ Bagé - RS - Brasil \\ wendellgasparoni@gmail.com, anaferreira@unipampa.edu.br, \\ \{cristina.genro,marcia.teixeira\}@embrapa.br
}

\begin{abstract}
This article introduces the mobile application H-Pasture, which uses linear regression models to provide tools for helping farmers and rural technicians perform grazing management activities using the pasture height metric. H-Pasture uses Google's Flutter/Dart technologies, makes forage weight estimations within the registered areas, builds reports, and produces recommendations for stocking rate under rotative or variable rate continuous pasture.
\end{abstract}

Resumo. Este trabalho apresenta o aplicativo móvel H-Pasture, que usa modelos de regressão linear para fornecer ferramentas que auxiliem produtores e técnicos rurais no manejo de pastagem por meio da métrica de altura do pasto. O aplicativo foi desenvolvido com a tecnologia Flutter/Dart e faz estimativas de massa de forragem nas áreas cadastradas, gera relatórios e produz recomendações para auxiliar no ajuste de carga em pastagens sob pastejo rotativo ou contínuo com taxa variável.

\section{Introdução}

Modelos de negócio baseados em pecuária extensiva dependem de uma boa estimativa de disponibilidade de alimento no pasto, para que a movimentação de animais entre potreiros possa ser realizada com precisão, garantindo bons resultados no processo de produção animal. Contudo, as pastagens são um ecossistema dinâmico e o manejo do pasto ainda é apontado por produtores e técnicos que utilizam esse ecossistema para a produção animal como desafiador, pois orientações e definições relativas à lotação dos piquetes e 
à definição do momento de entrada e saída dos animais nas áreas são pouco precisas [Genro e da Silveira 2018]. Frequentemente são observadas situações de superpastejo ou subpastejo, ambas acarretando em baixo desempenho animal, seja por produção vegetal aquém do potencial e degradação da pastagem, no primeiro caso, ou desperdício de forragem, no segundo [Hodgson 1990].

As pesquisas na área de manejo de pastagens têm trabalhado, principalmente, com dois conceitos de utilização do pasto: o primeiro diz respeito à oferta de forragem ou intensidade de pastejo, propondo o cálculo da oferta da quantidade de pasto existente na área em função de peso vivo animal (quilos de matéria seca ofertados para cada 100 quilos de peso vivo, ou \% PV), por um determinado período de tempo [Maraschin 2004]; o segundo é a interceptação luminosa, assumindo que o momento ideal para pastejo é quando o pasto está interceptando, em média, $95 \%$ da radiação que chega da atmosfera [Carnevalli et al. 2006, Barbosa et al. 2007]. Ambos os conceitos exigem treinamento das pessoas que vão conduzir as avaliações e equipamentos específicos, os quais dificilmente são encontrados em propriedades rurais.

Com o objetivo de construir um sistema de apoio ao manejo das pastagens, buscou-se um dos componentes da estrutura do pasto que apresentasse uma boa relação com a massa de forragem disponível para pastejo e que, ao mesmo tempo, fosse de mensuração simples. Diversos estudos com produção animal em pastagens cultivadas ou nativas têm apontado que a altura do pasto apresenta uma relação linear positiva com massa de forragem [Abramides et al. 1982, Trindade et al. 2007, Carvalho et al. 2010, Rosa et al. 2017, Genro e da Silveira 2018, Souza Filho et al. 2019]. Essa relação entre a altura e os componentes da estrutura do pasto, principalmente com a massa de forragem, viabiliza a utilização da altura como critério prático para definir o momento ideal de pastejo, bem como permite identificar a necessidade ou não de realização de ajustes de carga animal, visando estabelecer condições ótimas de utilização do pasto mediante os principais processos envolvidos no crescimento e utilização das plantas forrageiras sob pastejo.

Dados de pesquisa com forrageiras utilizadas nos sistemas de produção do Estado do Rio Grande do Sul têm reforçado a possibilidade do uso da altura como um método seguro para auxiliar o produtor no manejo do pasto da propriedade. Segundo Martins et al. (2015), a relação entre altura do pasto e a massa de forragem é uma importante ferramenta de manejo pois, ao controlar a altura, maneja-se indiretamente diferentes componentes do sistema. Adicionalmente, dentro da lógica de produção sustentável, tem sido possível observar que o manejo adequado do pasto contribui com serviços ambientais como a mitigação das emissões de gases de efeito estufa (GEE), por meio da redução das intensidades de emissão de metano por quilo de peso vivo produzido por área. Isso porque quando se usa a meta de altura adequada para manejo do pasto, os desempenhos individuais dos animais em pastejo são mais elevados [Savian et al. 2018] e mais carbono é sequestrado no solo [Franzluebbers e Stuedemann 2009].

Souza Filho et al. (2019) avaliaram o efeito de diferentes intensidades de pastejo por novilhos sobre o desempenho animal, o consumo de forragem e as emissões de $\mathrm{CH}_{4}$ no período de lotação de um sistema integrado soja-gado de corte no sul do Brasil. Os tratamentos foram diferentes, definidos pelas alturas-alvo de pastejo $(10,20,30 \mathrm{e}$ $40 \mathrm{~cm}$ ) em pastagem consorciada de aveia preta (Avena strigosa Schreb.) e azevém (Lo- 
lium multiflorum Lam.), sob lotação contínua. O manejo do pastejo afetou a utilização da forragem e, consequentemente, o desempenho animal e a emissão de metano. Individualmente, a emissão de metano e o desempenho dos animais apresentaram valores ótimos quando a altura do pasto foi manejada dentro de uma faixa de 23 a $30 \mathrm{~cm}$. Os dados agrupados mostraram um efeito linear positivo da intensidade do pastejo no ganho de peso vivo do animal por hectare e os custos ambientais associados ao uso da terra. O ganho de peso vivo aumentou em $90 \mathrm{~g} / \mathrm{ha} / \mathrm{dia}$ e as emissões de metano aumentaram em $500 \mathrm{~g}$ $\mathrm{CO}_{2}$ eq/ha/dia para cada centímetro de redução da altura do pasto alvo. Esses resultados mostram o impacto ambiental, em termos de emissões de GEE, que pode ser gerado sem otimização do manejo do pasto.

Genro e Silveira (2018) indicam a altura como um guia de manejo recomendado para auxiliar produtores e técnicos nas tomadas de decisão relativas ao uso das pastagens. Como a avaliação da altura é rápida, não destrutiva e de fácil entendimento, juntamente com o fato da altura ser um elo de ligação entre plantas, animais e o meio, incrementos significativos em produtividade podem ser alcançados a baixo custo. O objetivo deste trabalho é usar esses resultados para desenvolver um aplicativo que permita aos produtores e técnicos valerem-se das informações teóricas disponíveis para melhorar sua tomada de decisão quanto ao ajuste de carga em pastagens. O modelo implementado usa os valores para o RS, visto que podem haver diferenças regionais e da vegetação envolvida, mas pode ser adaptado para valores de outras localidades.

O restante do texto está organizado como se segue: a Seção 2 discute trabalhos com o mesmo objetivo encontrados na literatura, a Seção 3 apresenta as tecnologias utilizadas no desenvolvimento e as telas e funcionalidades mais relevantes do software e a Seção 4 conclui o texto, discutindo resultados e desenvolvimentos futuros.

\section{Trabalhos relacionados}

O desenvolvimento deste trabalho foi feito para dispositivos Android, visto que essa plataforma possui mais de $80 \%$ em participação de mercado de sistemas operacionais móveis no Brasil [StatCounter 2021]. Sendo assim, a busca por trabalhos correlatos foi orientada para a análise de aplicativos disponíveis na Google Play [Google 2021] e para aplicativos e outras ferramentas de software citadas em fontes especializadas. Com objetivos similares ao deste trabalho foram encontradas as seguintes aplicações: PastureMap [PastureMap 2021], Pasture.io [Pasture.io 2021], Grazing Wedge [University of Missouri 2021], Grazing Calculator [South Dakota State University 2021] e Grazing Calculator App [Robert Hathaway 2021].

Visto que grande parte das soluções encontradas são pagas, não foi possível a realização de testes com todas as funcionalidades, sendo que em alguns casos, como nas ferramentas Pasture.io e Grazing Calculator App, a análise foi feita baseado-se na documentação da ferramenta no site oficial. A Tabela 1 resume as ferramentas encontradas, sendo a primeira coluna o nome da ferramenta, a segunda a descrição da ferramenta e a terceira o custo para utilização.

Entre as ferramentas analisadas, PastureMap e o Pasture.io são as que disponibilizam o maior número de funcionalidades para os usuários, sendo que o Pasture.io coleta imagens de satélites para a estimativa de forragem, tornando este processo mais prático, porém, resultando em um custo mais alto para utilização. Por fim, considerando 
Tabela 1. Trabalhos Correlatos

\begin{tabular}{|l|l|c|}
\hline Ferramenta & Descrição & Custo \\
\hline PastureMap & $\begin{array}{l}\text { Ferramenta com recursos oferecidos para a gestão do manejo } \\
\text { de pastagem, possibilitando o cadastro de áreas, animais e } \\
\text { extração de relatórios para a tomada de decisão durante o ma- } \\
\text { nejo; o algoritmo utiliza os dados do Departamento de Agricul- } \\
\text { tura dos Estados Unidos, sem adequação a outras localidades. }\end{array}$ & paga \\
\hline Pasture.io & $\begin{array}{l}\text { Ferramenta com recursos oferecidos, com funcionalidades se- } \\
\text { melhantes ao PastureMap; a estimativa de forragem é calcu- } \\
\text { lada a partir de imagens extraídas de satélites, sem detalhes } \\
\text { sobre a técnica utilizada. }\end{array}$ & paga \\
\hline $\begin{array}{l}\text { Grazing } \\
\text { Wedge }\end{array}$ & $\begin{array}{l}\text { Desenvolvida e disponibilizada pela Universidade do Mis- } \\
\text { souri, possibilita o cadastro de propriedades rurais e suas áreas } \\
\text { de pastejo, calculando taxas de crescimento da pastagem e a } \\
\text { produção cumulativa de forragem no sistema de pastejo. }\end{array}$ & gratuita \\
\hline $\begin{array}{l}\text { Grazing Cal- } \\
\text { culator }\end{array}$ & $\begin{array}{l}\text { Calculadora disponibilizada pela Universidade do Estado de } \\
\text { Dakota do Sul por meio de um website, que a partir de dados } \\
\text { solicitados de entradas, como produção média de forragem, } \\
\text { tipo dos animas, qualidade da forragem, etc. calcula o total } \\
\text { de animais permitidos na área ou então a quantidade de dias } \\
\text { para manter os animais na área. }\end{array}$ & gratuita \\
\hline $\begin{array}{l}\text { Grazing Cal- } \\
\text { culator App }\end{array}$ & $\begin{array}{l}\text { Aplicativo disponibilizado para Android ou iOS que produz } \\
\text { diferentes cálculos sobre o processo de manejo, como quan- } \\
\text { tidade de dias para manter os animais na área, kg de matéria } \\
\text { seca por vaca por dia, entre outros cálculos. }\end{array}$ & paga \\
\hline
\end{tabular}

as soluções encontradas e comparando-as com os objetivos deste trabalho, recursos como a utilização de modelos matemáticos para tipos de forragens voltadas para a região da Campanha gaúcha, unidos à simplicidade de utilização e baixo custo, ainda tornam o $\mathrm{H}$ Pasture uma solução interessante para auxiliar produtores e técnicos rurais na tomada de decisão durante o manejo.

\section{O aplicativo H-Pasture}

O documento de requisitos da aplicação, inicialmente elaborado, engloba todos os requisitos funcionais e não funcionais que a aplicação deve conter, refletindo o método no qual se baseia. Os requisitos do aplicativo envolvem a sua utilização em acordo com as orientações sobre o manejo baseado em altura, que envolvem registros de medições de altura (dependendo da heterogeneidade da estrutura da vegetação), cálculo de massa de forragem disponível e recomendações específicas para evitar sub ou sobrepastejo. Para minimizar a quantidade de dados inseridos, o registro prévio de propriedades, suas áreas internas e tipo de vegetação em cada área é facultado ao usuário.

O levantamento das tecnologias adequadas para o desenvolvimento do aplicativo móvel, a fim de permitir uma maior flexibilidade de utilização para o usuário, destacou quatro abordagens: React Native [Facebook 2015], Ionic [Drifty 2013], Xamarin [Microsoft 2011] e Flutter [Google 2017]. A análise dos requisitos em comparação às 

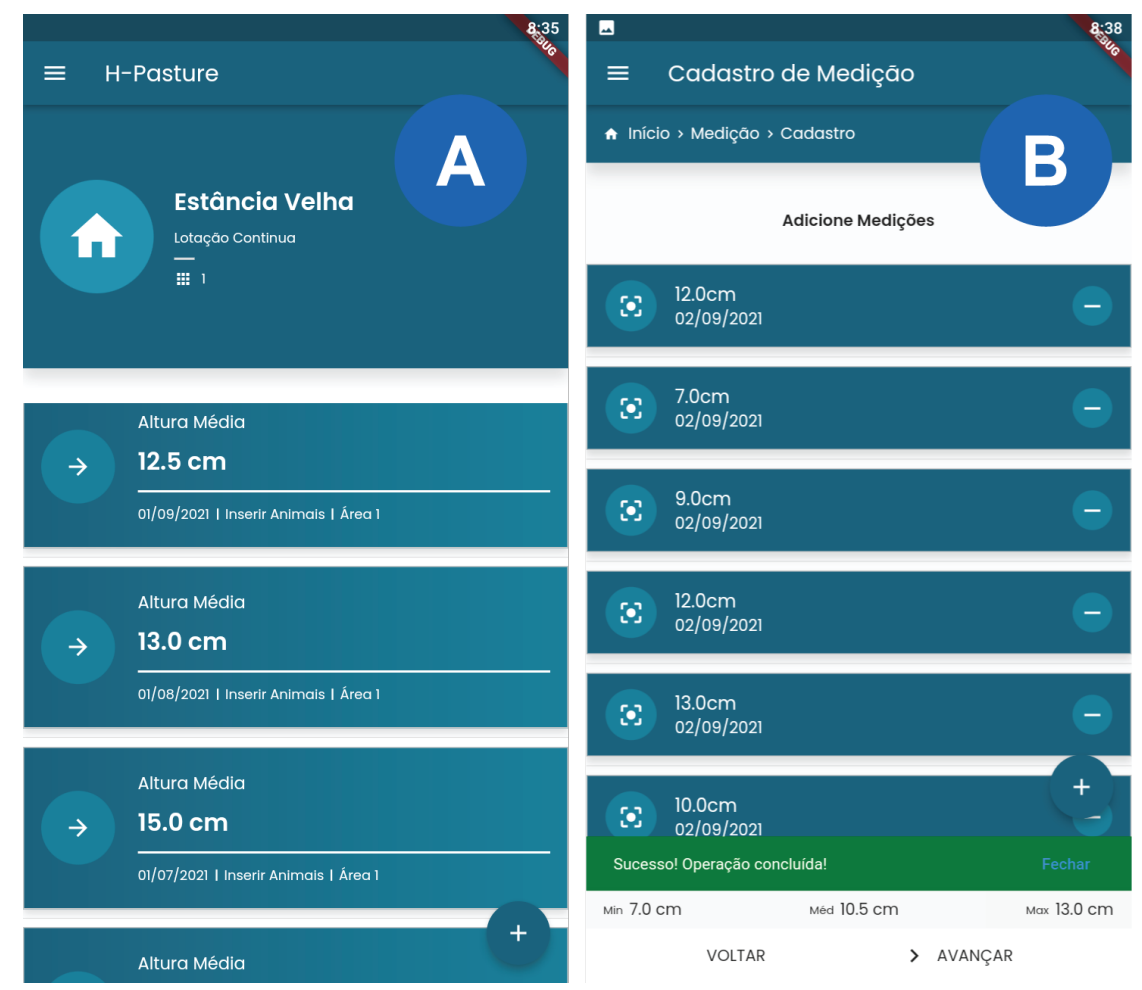

Figura 1. Tela inicial (A) e cadastro de medições (B)

tecnologias levantadas permitiu concluir que qualquer uma delas seria adequada. A tecnologia Flutter foi escolhida por pertencer a uma empresa de desenvolvimento sólida (Google) e pela sua curva de adoção pela comunidade estar aumentando consistentemente. A linguagem Dart, usada para desenvolvimento, contém construções que facilitam a portabilidade para diferentes sistemas operacionais e plataformas. Assim, ainda que o desenvolvimento tenha sido feito e testando no sistema operacionado Android, a migração para o sistema IOS pode ser feita sem alterações no código-fonte. Para possibilitar a utilização offline, foi utilizada uma base de dados local no dispositivo do usuário, com o banco de dados SQLite [D. Richard Hipp 2000].

O aplicativo contém informações sobre os tipos de pastagens mais comuns na região da Campanha gaúcha. A Tabela 2 apresenta os tipos de pastagem, a quantidade de massa seca estimada para cada centímetro de altura medido, recomendação de entrada e saída de animais, em função da altura do pasto em sistemas de pastejo rotativo e a indicação da revisão da taxa de lotação em áreas com pastejo contínuo.

Tabela 2. Indicações do aplicativo (cf. [Genro e da Silveira 2018])

\begin{tabular}{|c|c|c|c|c|}
\hline Pastagem & Massa (por cm) & \multicolumn{2}{|c|}{ Rotativo } & Contínuo \\
\cline { 3 - 5 } & & Entrada & Saída & Ajuste \\
\hline Azevém & $127 \mathrm{~kg} / \mathrm{ha}$ & $20 \mathrm{~cm}$ & 8 a $12 \mathrm{~cm}$ & 15 a $20 \mathrm{~cm}$ \\
Festuca & $165 \mathrm{~kg} / \mathrm{ha}$ & $20 \mathrm{~cm}$ & 6 a $12 \mathrm{~cm}$ & - \\
Capim-sudão & $63 \mathrm{~kg} / \mathrm{ha}$ & $60 \mathrm{~cm}$ & 15 a $20 \mathrm{~cm}$ & $30 \mathrm{a} 40 \mathrm{~cm}$ \\
Pasto nativo & $231,5 \mathrm{~kg} / \mathrm{ha}$ & $12 \mathrm{~cm}$ & $6 \mathrm{~cm}$ & 8 a $12 \mathrm{~cm}$ \\
\hline
\end{tabular}

Atualmente, as funcionalidades do aplicativo são: cadastro de uma ou mais pro- 
priedades rurais com diferentes áreas para pastejo, visualização do histórico de todas as medições já cadastradas, registros de medição de altura, visualização de dados e geração de relatório para auxiliar na tomada de decisão durante o manejo de pastagem.

A tela inicial, ilustrada na Figura 1A, apresenta ao usuário a propriedade rural atualmente selecionada junto a todo histórico de medições efetuadas em cada uma das áreas cadastradas da propriedade. O histórico das medições é apresentado ao usuário por meio de cartões contendo informações sintetizadas da medição: data da medição, nome da área, altura média do pasto e a ação sugerida (inserir ou retirar animais). A qualquer momento o usuário pode iniciar o processo de cadastro de novas medições por meio do botão de adição no canto inferior direito da Figura 1A. O cadastro da medição é feito selecionando a área desejada da propriedade rural e inserindo todas as medições coletadas em campo para a geração da média aritmética (Figura 1B). A média é aritmética porque o número de medições deve ser proporcional à área, levando em conta a heterogeneidade. A tela de cadastro de medição ainda fornece, para fins de referência, os valores parciais da medição até o momento: valor mínimo, valor máximo e a média aritmética das medidas inseridas até o momento.

A Figura 2 mostra a fase final do cadastro de medição da altura do pasto para uma área da propriedade rural, após a inserção das diferentes medições da altura. O aplicativo gera um relatório com ilustração (Figura 2A) das condições do pasto. Como observado, são geradas duas linhas em laranja para demarcar a faixa ótima de uso para os melhores ganhos em termos de produção animal e vegetal. A linha em vermelho demarca a altura média do pasto calculada a partir das medições informadas, e está nessa cor para indicar uma situação fora da faixa ideal de uso do pasto; caso contrário, a linha da média tem a cor azul. Nessa situação pode ocorrer perda de valor nutritivo aproveitado (acima do valor da altura de referência), ou ainda, comprometer a persistência do pasto e ser limitante ao consumo dos animais em pastejo (abaixo do valor da altura de referência).

Após a visualização do relatório apresentado na Figura 2A, o usuário pode gerar outro relatório com maior detalhamento. Ao clicar na seta da parte inferior da tela, o aplicativo apresenta o conteúdo da Figura 2B, com informações sobre limite de referência inferior, limite de referência superior, altura média do pasto e estimativa da massa de forragem disponível. Como pode ser observado, tanto o relatório simplificado da Figura 2A, quanto o detalhado da Figura 2B apresentam ao usuário, na parte inferior da tela, a ação sugerida. A lógica desta funcionalidade é implementada por meio do cruzamento de diferentes informações cadastradas. Como ilustrado na Figura 3A, durante o cadastramento da propriedade rural é informado o tipo de sistema de pastejo utilizado, podendo ser continuo ou rotativo. A Figura 3B ilustra o cadastramento de uma área para a propriedade rural. Nesse cadastro é informado o tipo da forragem da área, podendo ser forragem nativa, festuca, capim-sudão, ou qualquer outra previamente cadastrada. Estas informações, junto à média da altura do pasto coletada durante o registro de medição, são cruzadas e analisadas conforme a Tabela 2. Após a análise, se for observado que a altura está acima do limite máximo, é recomendado um ajuste de carga com a inserção de animais na área. $\mathrm{O}$ aplicativo também informa qual o peso vivo por hectare que pode ser inserido, em função do excedente calculado de massa de forragem. Caso a altura esteja abaixo do limite mínimo é sugerido um ajuste através da remoção de animais da área. Os dados informados nas figuras são dados gerados aleatoriamente, para fins de apresentação do 


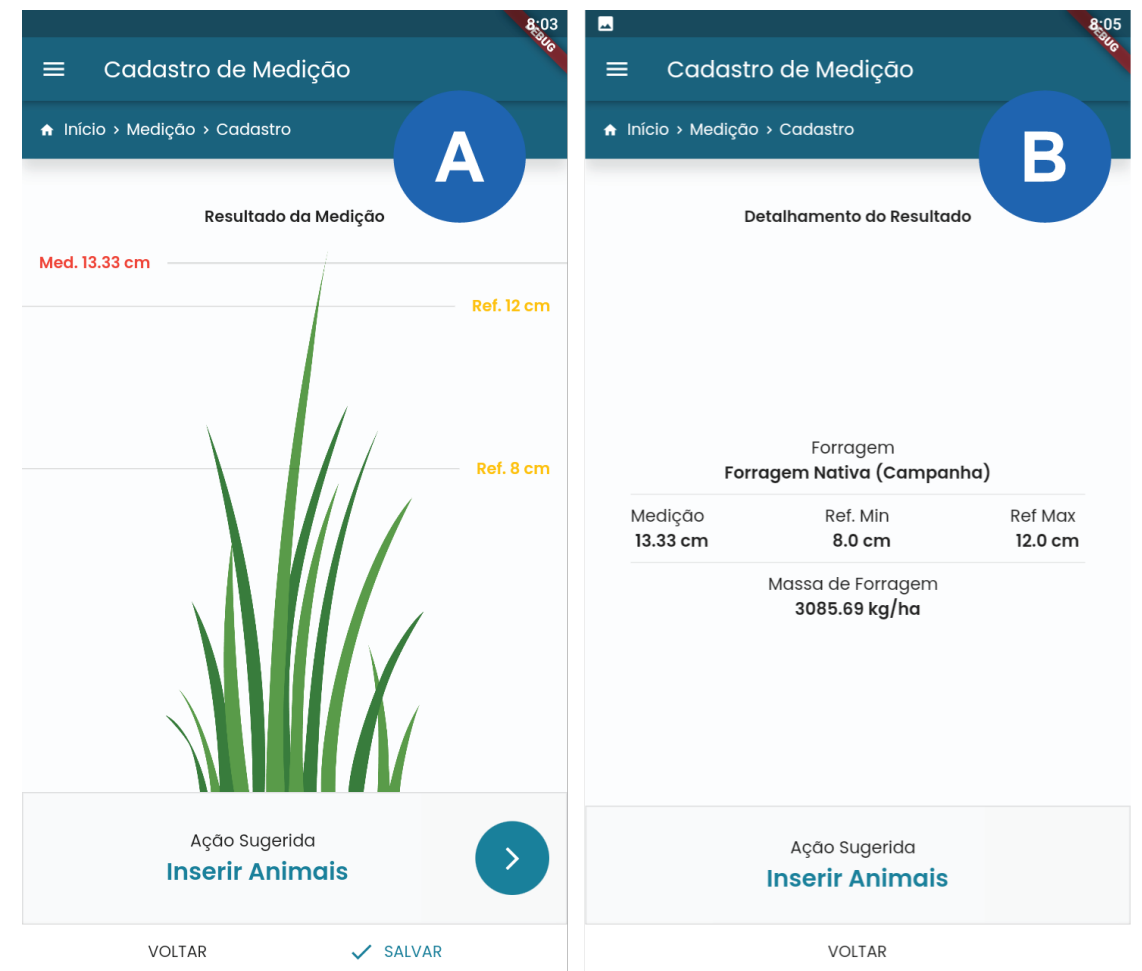

Figura 2. Telas de relatórios

aplicativo neste trabalho.

\section{Conclusão}

O manejo das pastagens e ajuste de lotação com base na altura do pasto é uma técnica simples que não requer equipamento ou pessoal especializado, ao contrário de outras técnicas de predição de massa de forragem. Ainda que bastante simples, a literatura aponta que é uma técnica que produz boas estimativas.

O H-Pasture é uma aplicação para tornar o processo de manejo de pastagem mais simples para produtores e técnicos rurais. Baseado nos modelos matemáticos e nas orientações publicadas em [Genro e da Silveira 2018] a aplicação fornece, ao produtor ou técnico, ferramentas didáticas para o manejo. A partir do cadastro de medições, a aplicação gera o cálculo de massa de forragem, ajudando o usuário na tomada de decisão durante o manejo de pastagem. O uso da altura do pasto como critério de manejo possibilita um ajuste mais preciso para evitar situações de sobre ou subpastejo, com a vantagem do baixo custo. $\mathrm{O}$ aplicativo $\mathrm{H}$-Pasture facilita ainda mais o trabalho, visto que apresenta os cálculos e a recomendação de manejo de forma automática, funcionando sem necessidade de conexão à internet.

O H-Pasture somente possui dados para quatro tipos de pastagens, com dados voltados à região da Campanha gaúcha. Essa restrição não é permanente e o código pode ser facilmente estendido para adicionar mais dados de outras regiões do país, com culturas também pertinentes a essas regiões. Dados de GPS do próprio celular podem ser usados para orientar as recomendações, a partir da inserção de uma base de dados sobre culturas que possa ser acessada via internet. 

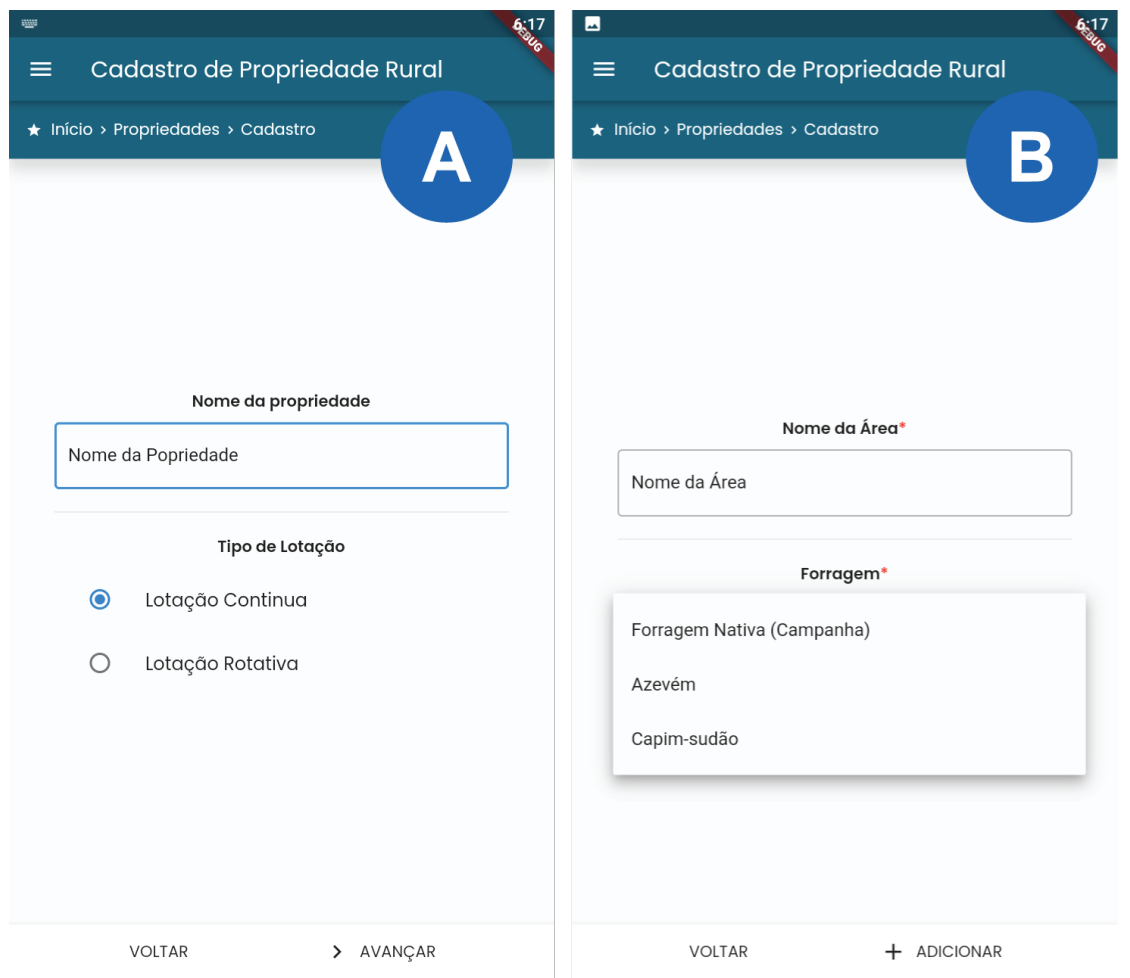

Figura 3. Telas de cadastros

A orientação sobre o processo de medição e sua frequência está sendo colocada no código do aplicativo. Também serão adicionados alertas para informar ao usuário o momento de coletar novas medidas de altura do pasto. A informação será dependente das estações do ano e do tipo de sistema de pastejo usado. Atualmente, o aplicativo está na fase de testes de aceitação, com o feedback de usuários especializados. Os testes com os usuários finais serão realizados nos próximos meses, quando o aplicativo será colocado à disposição da comunidade.

\section{Referências}

Abramides, P., Alcântara, P., Stafuzza, J., Foschine, A., Dower, J. (1982). Estimativa da quantidade de forragem em pastagens de capins prostrados tropicais, através da medida da altura média da vegetação. 20(1):17-41.

Barbosa, R. A., Nascimento Jr, D., Euclides, V. P. B., Silva, S. C., Zimmer, A. H., Torres Jr, R. A. A. (2007). Capim-tanzânia submetido a combinações entre intensidade e frequiência de pastejo. Pesquisa Agropecuária Brasileira, 42(3):329-340.

Carnevalli, R. A., Silva, S. C., Bueno, A. A. O., Uebele, M. C., Bueno, F., Hodgson, J., Silva, G. N., Moraes, J. P. G. (2006). Herbage production and grazing losses in panicum maximum cv. mombaça under four grazing managements. Tropical Grasslands, 40:165-176.

Carvalho, P. C. F., Rocha, L. M., Baggio, C., Macari, S., Kunrath, T. R., Moraes, A. (2010). Característica produtiva e estrutural de pastos mistos de aveia e azevém manejados em quatro alturas sob lotação continua. Revista Brasileira de Zootecnia, 39(9):1857-1865. 
D. Richard Hipp (2000). SQLite. Disponível em: https: //www. sqlite.org//. Acesso em: setembro 2021.

Drifty (2013). Ionic. Disponível em: https://ionicframework.com/. Acesso em: setembro 2021.

Facebook (2015). React native. Disponível em: https://reactnative.dev/. Acesso em: setembro 2021.

Franzluebbers, A. J. Stuedemann, J. A. (2009). Soil-profile organic carbon and total nitrogen during 12 years of pasture management in the southern piedmont usa. Agric. Ecosyst. Environ., 129:28-36.

Genro, T. C. M. da Silveira, M. C. T. (2018). Uso da altura para ajuste de carga em pastagens. Comunicado Técnico 101, Embrapa Pecuária Sul.

Google (2017). Flutter. Disponível em: https://flutter.dev/. Acesso em: setembro 2021.

Google (2021). Google play. Disponível em: https://play.google.com/store. Acesso em: 11 out. 2021.

Hodgson, J. (1990). Grazing management: science into practice. John Willey, Longman Scientific and Technical, New York.

Maraschin, G. E. (2004). Estratégias para valorizar sistemas pastoris sob a ótica de políticas de segurança alimentar, bem estar animal e social, pages 67-83. Number 20. GRUPO CAMPOS, Salto.

Martins, A. P., aise Robinson Kunrath, Anghinoni, I., de Faccio Carvalho, P. C. (2015). Integração Soja-Bovinos de Corte no Sul do Brasil. Grupo de Pesquisa em Sistema Integrado de Produção Agropecuária, UFRGS, Porto Alegre.

Microsoft (2011). Xamarin. Disponível em: https : / xamarin.com/. Acesso em: setembro 2021.

Pasture.io (2021). Pasture.io. Disponível em: https://pasture.io/. Acesso em: 11 out. 2021.

PastureMap (2021). Pasturemap. Disponível em: https://pasturemap.com/. Acesso em: 11 out. 2021.

Robert Hathaway, L. H. (2021). Grazing calculator. Disponível em: https://www.grazingcalculator.com/. Acesso em: 11 out. 2021.

Rosa, F. Q., Bremm, C., Machado, D. R. (2017). Efeito da oferta de forragem na estrutura do pasto, pages 23-25. UFRGS, Porto Alegre.

Savian, J. V., Schons, R. M. T., Marchi, D. E., de Freitas, T. S., Silva Neto, G. F., Mezzalira, J. C., de Faccio Carvalho, P. C. (2018). Rotatinuous stocking: a grazing management innovation that has high potential to mitigate methane emissions by sheep. $J$. Clean. Prod., 186:602-608.

South Dakota State University (2021). Grazing calculator. Disponível em: https://extension.sdstate.edu/grazing-calculator. Acesso em: 11 out. 2021.

Souza Filho, W., Nunes, P. A. d. A., Barro, R. S., Kunrath, T. R., Almeida, G. M., Genro, T. C. M., Bayer, C., de Faccio Carvalho, P. C. (2019). Mitigation of enteric 
methane emissions through pasture management in integrated crop-livestock systems: trade-offs between animal performance and environmental impacts. J. Clean. Prod., 213:968-975.

StatCounter (2021). Mobile operating system market share brazil. Disponível em: https://gs.statcounter.com/os-market-share/mobile/brazil/\#monthly-202010-202110.

Acesso em: 11 out. 2021.

Trindade, J. K. et al. (2007). Composição morfológica da forragem consumida por bovinos de corte durante o rebaixamento do capim-marandu submetido a estratégias de pastejo rotativo. Pesquisa Agropecuária Brasileira, 42(6):883-890.

University of Missouri (2021). Grazing wedge. Disponível em: https://grazingwedge.missouri.edu/. Acesso em: 11 out. 2021. 